Korean J. Math. 19 (2011), No. 4, pp. 403-407

\title{
REMARKS ON A GOLDBACH PROPERTY
}

\author{
SUN JU JANG
}

\begin{abstract}
In this paper, we study Noetherian Boolean rings. We show that if $R$ is a Noetherian Boolean ring, then $R$ is finite and $R \simeq\left(\mathbb{Z}_{2}\right)^{n}$ for some integer $n \geq 1$. If $R$ is a Noetherian ring, then $R / J$ is a Noetherian Boolean ring, where $J$ is the intersection of all ideals $I$ of $R$ with $|R / I|=2$. Thus $R / J$ is finite, and hence the set of ideals $I$ of $R$ with $|R / I|=2$ is finite. We also give a short proof of Hayes's result : For every polynomial $f(x) \in \mathbb{Z}[x]$ of degree $n \geq 1$, there are irreducible polynomials $g(x)$ and $h(x)$, each of degree $n$, such that $g(x)+h(x)=f(x)$.
\end{abstract}

All rings are assumed to be commutative rings with identity. We use the term dimension of $R$, denoted $\operatorname{dim} R$, to refer to the Krull dimension of $R$. A ring $R$ is called von Neumann regular if for each $x$ in $R$, there exists $y$ in $R$ such that $x=x y x$. It is well known that $R$ is von Neumann regular if and only if $R$ is zero-dimensional and reduced if and only if $R_{P}$ is a field for each $P \in \operatorname{Spec}(R)$ if and only if each ideal of $R$ is a radical ideal if and only if each principal ideal of $R$ is idempotent [4, Theorem 3.1]. In particular, $\operatorname{dim} R=0$ if and only if $R / \operatorname{nil}(R)$ is von Neumann regular (where $\operatorname{nil}(R)$ is the nilradical of $R$ ) if and only if a power of each principal ideal of $R$ is idempotent - that is, if and only if, for each $x \in R$, there exists $n(x) \in \mathbb{Z}^{+}$and $y \in R$ such that $x^{n(x)}=y x^{n(x)+1}[4$, Theorem 3.4]. The class of von Neumann regular rings is closed under taking homomorphic images, quotient rings, and arbitrary products [4, Result 3.2].

$R$ is called a Boolean ring if every element is idempotent. It is well known that $R$ is a Boolean ring if and only if $R_{M}$ is isomorphic to $\mathbb{Z}_{2}$ for each maximal ideal $M$ of $R$. A Boolean ring is a von Neumann regular

Received October 13, 2011. Revised November 21, 2011. Accepted November 30, 2011.

2000 Mathematics Subject Classification: 13B25, $13 \mathrm{E} 05$.

Key words and phrases: Noetherian ring, Boolean ring, von Neumann regular ring, Goldbach property. 
ring with $x=x 1 x$. It is known that $R / \operatorname{nil}(R)$ is Boolean if and only if $\operatorname{dim} R=0$ and for each maximal ideal $M$ of $R, R / M \simeq \mathbb{Z}_{2}$ if and only if given $x \in R$, there exists a natural number $n$ with $x^{n}(1+x)^{n}=0$ [1, Theorem 5].

In this paper, we study Noetherian Boolean rings. We show that if $R$ is a Noetherian Boolean ring, then $R$ is finite and $R \simeq\left(\mathbb{Z}_{2}\right)^{n}$ for some integer $n \geq 1$. If $R$ is a Noetherian ring, then $R / J$ is a Noetherian Boolean ring, where $J$ is the intersection of all ideals of $\mathcal{I}_{2}=\{I \mid I$ is an ideal of $R$ and $|R / I|=2\}$. Thus $R / J$ is finite, and hence the set $\mathcal{I}_{2}$ is finite. We also give a short proof of Hayes's result using Chinese Remainder Theorem for rings.

For future reference, we include a result from [4, Theorem 3.1(4)].

Lemma 1. If $R$ is a Boolean ring, then $M^{2}=M$ for each ideal $M$ of $R$.

Proof. If $x \in M$, then $x=x^{2} \in M^{2}$. Hence $M \subseteq M^{2}$, and thus $M=M^{2}$.

Let $I$ and $J$ be ideals of $R$. Recall that $I$ and $J$ are comaximal if $I+J=R$. Suppose that $I$ and $J$ are comaximal. Then there exist $a \in I$ and $b \in J$ such that $a+b=1$. For any integer $m, n(\geq 1)$, $1=(a+b)^{m+n}$ and $(a+b)^{m+n} \in I^{m}+J^{n}$; so $I^{m}+J^{n}=R$, and hence $I^{m}$ and $J^{n}$ are also comaximal [7, Lemma 4].

For future reference, we include the Chinese Remainder Theorem $[3$, Section 7.6].

LEMma 2. (Chinese Remainder Theorem) Let $I_{1}, I_{2}, \ldots, I_{n}$ be ideals of $R$. The map $R \rightarrow R / I_{1} \times R / I_{2} \times \cdots \times R / I_{n}$ defined by $r \mapsto\left(r+I_{1}, r+\right.$ $\left.I_{2}, \ldots, r+I_{n}\right)$ is a ring homomorphism with kernel $I_{1} \cap I_{2} \cap \cdots \cap I_{n}$. If each ideals $I_{i}, I_{j}(i \neq j)$ are comaximal, then the map is surjective and $I_{1} \cap I_{2} \cap \cdots \cap I_{n}=I_{1} I_{2} \cdots I_{n}$, so $R /\left(I_{1} I_{2} \cdots I_{n}\right) \simeq R /\left(I_{1} \cap I_{2} \cap \cdots \cap I_{n}\right) \simeq$ $R / I_{1} \times R / I_{2} \times \cdots \times R / I_{n}$.

If $R$ is a finite Boolean ring, then $R \simeq \mathbb{Z}_{2} \times \mathbb{Z}_{2} \times \cdots \times \mathbb{Z}_{2}$ (c.f. [3, Exercise 2, p. 267]). We next show that a Noetherian Boolean ring $R$ is finite with $R \simeq\left(\mathbb{Z}_{2}\right)^{n}$ for some integer $n \geq 1$.

Theorem 3. Let $R$ be a Boolean ring. 
(1) $R$ is zero-dimensional reduced and for each maximal ideal $M$ of $R, R / M \simeq \mathbb{Z}_{2}$.

(2) If $R$ is Noetherian, then $R$ is a finite ring with $R \simeq\left(\mathbb{Z}_{2}\right)^{n}$ for some integer $n \geq 1$.

Proof. (1) Suppose that $\operatorname{dim} R>0$. Then there are primes $P \subsetneq M$. Let $x \in M-P$. Then $x=x^{2}$, and so $x(x-1)=0 \in P$. Since $x \notin P$, we have $x-1 \in P \subseteq M$. But $x \in M$, so $1 \in M$, a contradiction. Clearly $\operatorname{nil}(R)=\{0\}$. Let $M$ be a maximal ideal of $R$. Then $R / M$ is a field and a Boolean ring; so $R / M \simeq \mathbb{Z}_{2}$.

(2) Suppose that $R$ is Noetherian. Then since, each ideal of $R$ contains a product of prime ideals of $R$ [3, Corollary 22, p. 685], we have $(0)=P_{1}^{r_{1}} P_{2}{ }^{r_{2}} \cdots P_{n}^{r_{n}}$. By Lemma 1 , each $P_{i}^{r_{i}}=P_{i}$; so (0) $=P_{1} P_{2} \cdots P_{n}$ and $P_{1}, P_{2}, \ldots, P_{n}$ are distinct. Since the ideals $P_{i}$ and $P_{j}$ with $i \neq j$ are comaximal, the map $R \rightarrow R / P_{1} \times R / P_{2} \cdots \times$ $R / P_{n}, r \mapsto\left(r+P_{1}, r+P_{2}, \ldots, r+P_{n}\right)$ is an epimorphism with kernel $P_{1} \cap P_{2} \cap \cdots \cap P_{n}=P_{1} P_{2} \cdots P_{n}=\{0\}$ by Lemma 2. Hence $R \simeq$ $R /\{0\} \simeq R / P_{1} \times R / P_{2} \times \cdots \times R / P_{n}$. Now, each $R / P_{i} \simeq \mathbb{Z}_{2}$ by (1). Hence $R \simeq\left(\mathbb{Z}_{2}\right)^{n}$ for some integer $n \geq 1$.

Corollary 4. (c.f., [7, Lemma 7], [9, Proposition 13]) Let $R$ be a ring and let

$$
\mathcal{I}_{2}=\{I \mid I \text { is an ideal of } R \text { and }|R / I|=2\} \text {. }
$$

Let $J$ be the intersection of all ideals in $\mathcal{I}_{2}$. Then $R / J$ is a Boolean ring. Moreover, if $R$ is Noetherian, then $R / J$ is a finite ring with $|R / J|=2^{n}$ for some integer $n \geq 1$ and $\mathcal{I}_{2}$ is finite.

Proof. Let $x \in R$. For each $I \in \mathcal{I}_{2}$, we have $x^{2}-x \in I$. Thus for each $x \in R, x^{2}-x \in \bigcap\left\{I \mid I \in \mathcal{I}_{2}\right\}=J$. Therefore $R / J$ is a Boolean ring. In particular, if $R$ is Noetherian, then $R / J$ is Noetherian, and so by Theorem $3, R / J$ is a finite ring with $|R / J|=2^{n}$ for some integer $n \geq 1$. Hence $\{I / J|| R / I \mid=2\}$ is finite. Since the map $I \rightarrow I / J$ is injective, $\mathcal{I}_{2}$ is finite.

Let $R$ be a Noetherian ring and let $\mathcal{I}_{n}=\left\{I_{a}\right\}_{a \in \Lambda}$, where $\left|R / I_{a}\right|=n$. Define $J=\bigcap_{a \in \Lambda} I_{a}$. Then $R / J$ can be imbedded in $\prod_{a \in \Lambda}\left(R / I_{a}\right)$. Then $R / J$ is zero-dimensional Noetherian and hence Artinian. Hence 
$J=\bigcap I_{a}$ has a finite subintersections, so $R / J$ is imbedded in $\prod_{i=1}^{k}\left(R / I_{a_{i}}\right)$, a ring of cardinality $n^{k}$. Therefore $R / J$ is finite and hence $\left\{I_{a} / J\right\}_{a \in \Lambda}$ is finite. Since the map $I_{a} \rightarrow I_{a} / J$ is injective, $\mathcal{I}_{n}=\left\{I_{a}\right\}_{a \in \Lambda}$ is finite $[5$, Result 3].

D. Hayes [6] was the first to observe and prove the following polynomial analogue of the celebrated Goldbach conjecture:

Theorem 5. For every polynomial $f(x) \in \mathbb{Z}[x]$ of degree $n \geq 1$, there are irreducible polynomials $g(x)$ and $h(x)$, each of degree $n$, such that $g(x)+h(x)=f(x)$.

To prove Theorem 5, Hayes used the following [6, Lemma]: if $p$ and $q$ are distinct odd primes, then there exist integers $c$ and $d$ such that $p c+q d=1, p \nmid c$, and $q \nmid d$. Also, Hayes pointed out that more general theorem whenever $R$ is a principal ideal domain with infinitely many maximal ideals. In [7], P. Pollack showed the case that $R$ is a Noetherian domain with infinitely many maximal ideals: Suppose that $R$ is an integral domain which is Noetherian and has infinitely many maximal ideals. Then every element of $R[x]$ of degree $n \geq 1$ can be written as the sum of two irreducibles of degree $n$. He used distinct maximal ideals $P$ and $Q$ such that $(1) P^{2} \neq P$ and $Q^{2} \neq Q$, (2) $|R / P|,|R / Q|>2[7$, Theorem 5]. Noetherian condition guarantees that $\mathcal{I}_{2}=\{I \mid I$ is an ideal of $R$ and $|R / I|=2\}$ is finite by Corollary 4, and if $M$ is maximal, then $M^{2} \neq M$ [7, Lemma 6]. Also, in [8], F. Saidak gives a short proof of Hayes's result.

In order to prove Theorem 5, we recall the remarkable criterion of Eisenstein [2].

LEMma 6. (Eisenstein's criterion) If, in the integral polynomial $a_{0} x^{n}+a_{1} x^{n-1}+\cdots+a_{n}$, all of the coefficients except $a_{0}$ are divisible by a prime $p$, but $a_{n}$ is not divisible by $p^{2}$, then the polynomial is irreducible.

Proof of Theorem 5. Write $f(x)=m_{0} x^{n}+m_{1} x^{n-1}+\cdots+m_{n}$. Choose distinct odd primes $p$ and $q$ which do not divide either of $m_{0}$ and $m_{n}$. Let $R=\mathbb{Z}, p R=P$, and $q R=Q$. Since $P$ and $Q$ are comaximal, $P^{2}$ and $Q^{2}$ are also comaximal. Therefore the two maps $R \rightarrow R / P \times R / Q, r \mapsto(r+P, r+Q)$ and $R \rightarrow R / P^{2} \times R / Q^{2}$, $r \mapsto\left(r+P^{2}, r+Q^{2}\right)$ are surjective homomorphisms by Lemma 2 . Choose $\alpha \notin P$ and $\beta \notin Q$. Let $a_{0}$ be a preimage of $\left(\alpha+P, m_{0}-\beta+Q\right)$ 
under $R \rightarrow R / P \times R / Q$. Set $b_{0}=m_{0}-a_{0}$. Then $a_{0} \notin P$ and $b_{0} \notin Q$. Also, for $i(0<i<n)$, let $a_{i}$ be a preimage of $\left(0+P, m_{i}+Q\right)$ under $R \rightarrow R / P \times R / Q$. Set $b_{i}=m_{i}-a_{i}$. Then $a_{i} \in P$ and $b_{i} \in Q$. Finally, let $a_{n}$ be a preimage of $\left(p+P^{2}, m_{n}-q+Q^{2}\right)$ under $R \rightarrow R / P^{2} \times R / Q^{2}$. Set $b_{n}=m_{n}-a_{n}$. Then we have $a_{n} \in P, a_{n} \notin P^{2}, b_{n} \in Q$, and $b_{n} \notin Q^{2}$. If $g(x)=a_{0} x^{n}+a_{1} x^{n-1}+\cdots+a_{n}$ and if $h(x)=b_{0} x^{n}+b_{1} x^{n-1}+\cdots+$ $b_{n}$, then $f(x)=g(x)+h(x)$. Lemma 6 says that $g(x)$ and $h(x)$ are irreducible polynomials.

Remark 7. (cf. [6, Theorem 1]) As the same notation above, Hayes choose $a_{n}{ }^{\prime}$ and $b_{n}{ }^{\prime}$ such that $p a_{n}{ }^{\prime}+q b_{n}{ }^{\prime}=m_{n}$, but $p \nmid a_{n}{ }^{\prime}$ and $q \nmid b_{n}{ }^{\prime}$ by $\left[6\right.$, Lemma]. Set $a_{n}=p a_{n}{ }^{\prime}$ and $b_{n}=q b_{n}{ }^{\prime}$. Then $m_{n}=a_{n}+b_{n}$, $p\left|a_{n}, p^{2} \nmid a_{n}, q\right| b_{n}$, and $q^{2} \nmid b_{n}$.

Acknowledgement. We would like to thank referee for several useful suggestions.

\section{References}

[1] D.D. Anderson, Generalizations of Boolean rings, Boolean-like rings and von Neumann regular rings, Comment. Math. Univ. St. Pauli 35 (1986), 69-76.

[2] D. Cox, Why Eisenstein proved the Eisenstein criterion and why Schönemann discovered it first, Amer. Math. Monthly 118 (2011), 3-21.

[3] D. Dummit and R. Foots, Abstract Algebra, 3rd ed., John Wiley, Hoboken, NJ, 2004.

[4] R. Gilmer, Background and preliminaries on zero-dimensional rings, Lect. Notes Pure Appl. Math. 171 (1994), 1-13.

[5] R. Gilmer, Zero-dimensionality and products of commutative rings, Lect. Notes Pure Appl. Math. 171 (1994), 15-25.

[6] D. Hayes, A Goldbach theorem for polynomials with integral coefficients, Amer. Math. Monthly 72 (1965), 45-46.

[7] P. Pollack, On polynomial rings with a Goldbach property, Amer. Math. Monthly 118 (2011), 71-77.

[8] F. Saidak, On Goldbach's conjecture for integer polynomials, Amer. Math. Monthly 113 (2006), 541-545.

[9] P. Samuel, About Euclidean rings, J. Algebra 19 (1971), 282-301.

Department of Mathematics

Inha University

Incheon 402-751 Korea

E-mail: jangsj@inha.edu 